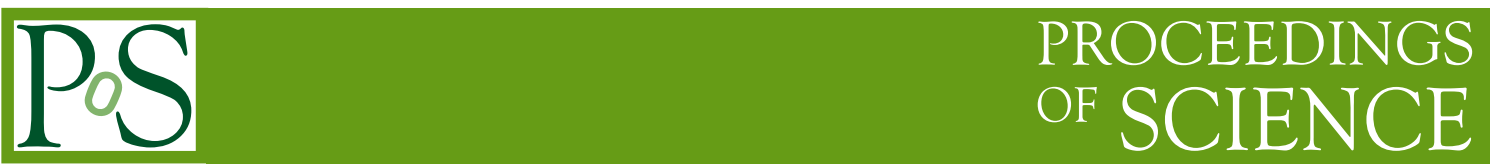

\title{
Performance of the ALICE RPC-based muon trigger system
}

\author{
Francesco Bossù and Martino Gagliardi* \\ Università degli Studi and Sezione INFN Torino, Italy \\ E-mail: bossuato.infn.it \\ E-mail: gagliardeto.infn.it
}

\section{for the ALICE Collaboration}

The ALICE muon spectrometer is equipped with a trigger system made of four RPC planes, arranged in two stations with two detection planes each. The aim of the system is to provide single and di-muon triggers with suitable transverse momentum selection, optimised for the physics of quarkonia and open heavy flavour.

In the first two years of data-taking at the LHC, the 72 RPCs were operated in highly saturated avalanche mode in both $\mathrm{pp}$ and $\mathrm{Pb}-\mathrm{Pb}$ collisions. The integrated number of hits is about $13 \mathrm{Mhit} / \mathrm{cm}^{2}$ on average and $35 \mathrm{Mhit} / \mathrm{cm}^{2}$ for the most exposed detectors.

The performance of the RPCs and of the system as a whole is discussed, with particular focus on their stability in time.

XI Workshop on Resistive Plate Chambers and Related Detectors - RPC2012,

February 5-10, 2012

INFN Laboratori Nazionali di Frascati Italy

\footnotetext{
* Speaker.
} 


\section{The ALICE muon trigger system}

The ALICE [1] experiment at the LHC has as its primary goal the study of nuclear matter at very high temperatures and energy densities, and the transition to a deconfined state of matter, known as Quark Gluon Plasma (QGP). This is done via the analysis of ultra-relativistic heavy-ion collisions at the LHC. Proton-proton physics is also included in the ALICE program, both as a reference for observables measured in heavy-ion collisions and as a per se field of study.

Heavy flavour production is sensitive to the properties of QGP. In the ALICE muon spectrometer, heavy flavoured mesons are detected via their muonic and semi-muonic decays [2]. The spectrometer is equipped with a dedicated muon trigger system featuring 72 RPCs arranged in two stations, located, respectively, at a distance of 16 and $17 \mathrm{~m}$ from the interaction point. Each station is made of two detection planes with 18 RPCs each. The total active area of the system is about $140 \mathrm{~m}^{2}$. The total number of electronics channels is about 21000 . The spatial information provided by the RPCs is used to perform a selection on the muon transverse momentum $\left(\mathrm{p}_{\mathrm{T}}\right)$, via the deviation with respect to the trajectory of an infinite momentum track originated at the interaction point. The system is able to deliver single and di-muon (unlike- and like-sign) triggers. For each of these signals, two different $\mathrm{p}_{\mathrm{T}}$ cuts can be handled simultaneously, for a total of six trigger signals evaluated and delivered to the ALICE trigger processor at a frequency of $40 \mathrm{MHz}$ and with a latency of about $800 \mathrm{~ns}$. More details about the trigger algorithm and electronics are given in a separate contribution to this Conference [3].

\section{Operating conditions in $\mathbf{2 0 1 0 - 1 1}$}

In 2010 and 2011, ALICE took pp collision data at $\sqrt{s}=7 \mathrm{TeV}$ (8 months/year) ${ }^{1}$ and in $\mathrm{Pb}-\mathrm{Pb}$ collisions at $\sqrt{s_{N N}}=2.76 \mathrm{TeV}$ (1 month/year). In 2010, the typical pp luminosity was about $10^{29} \mathrm{~cm}^{-2} \mathrm{~s}^{-1}$, corresponding to a single muon trigger rate of about $100 \mathrm{~Hz}$, with a $\mathrm{p}_{\mathrm{T}}$ cut of $0.5 \mathrm{GeV} / \mathrm{c}$. The $\mathrm{Pb}-\mathrm{Pb}$ luminosity was about $10^{25} \mathrm{~cm}^{-2} \mathrm{~s}^{-1}$. Given the low luminosity, no dedicated muon trigger in $\mathrm{Pb}-\mathrm{Pb}$ collisions was needed. However, the muon trigger data was read out and used for offline matching [4]. In 2011, the pp luminosity was about $2 \times 10^{30} \mathrm{~cm}^{-2} \mathrm{~s}^{-1}$, corresponding to a single muon trigger rate of about $500 \mathrm{~Hz}$ and to a di-muon trigger rate of about $20 \mathrm{~Hz}$, with a $\mathrm{p}_{\mathrm{T}}$ cut of $1 \mathrm{GeV} / \mathrm{c}$. The $\mathrm{Pb}-\mathrm{Pb}$ luminosity was about $3 \times 10^{26} \mathrm{~cm}^{-2} \mathrm{~s}^{-1}$, corresponding to a single muon trigger rate of about $500 \mathrm{~Hz}$ and to a di-muon trigger rate of about $200 \mathrm{~Hz}$, with a $\mathrm{p}_{\mathrm{T}}$ cut of $1 \mathrm{GeV} / \mathrm{c}$.

The fraction of operational Front-End Electronics (FEE) channels was 99.7\% at the end of the 2011 data-taking. The average number of trigger errors per event (evaluated by comparing the online and offline trigger decision) is about $10^{-4}\left(10^{-3}\right)$ in $\mathrm{pp}(\mathrm{Pb}-\mathrm{Pb})$ collisions. The performance of the trigger system in $\mathrm{Pb}-\mathrm{Pb}$ collisions is discussed in detail in a separate contribution to this Conference [5].

\section{The ALICE muon trigger RPCs}

The ALICE muon trigger detectors are single gap RPCs, with low resistivity $\left(3 \div 9 \times 10^{9} \Omega \mathrm{cm}\right)$

\footnotetext{
${ }^{1}$ In 2011, data was also taken for a few days in pp collisions at $\sqrt{s}=2.76 \mathrm{TeV}$
} 
bakelite electrodes. They are operated in highly saturated avalanche mode, with the following gas mixture: $89.7 \% \mathrm{C}_{2} \mathrm{H}_{2} \mathrm{~F}_{4}, 10 \% \mathrm{C}_{4} \mathrm{H}_{10}, 0.3 \% \mathrm{SF}_{6}$. The gas relative humidity is kept at $37 \%$, in order to prevent alterations in the bakelite resistivity [6,7]. The individual RPC areas range from $72 \times 223 \mathrm{~cm}^{2}$ to $76 \times 292 \mathrm{~cm}^{2}$. The signal is picked up inductively on both sides of the detector by means of orthogonal (X and Y) copper strips. Strips with pitch of 1,2 and $4 \mathrm{~cm}$ and length ranging from 17 to $72 \mathrm{~cm}$ are employed; areas closer to the beam pipe have the finest segmentation. The RPC signal is discriminated in the FEE without pre-amplification [8]. The signal amplitude threshold can be separately adjusted for each RPC via the Detector Control System (DCS); it is set to $7 \mathrm{mV}$ for most RPCs.

The operating high voltage was optimised for each RPC with cosmic data, and fine-tuned with early pp data [6,9]; typical values are around $10.3 \mathrm{kV}$. Operating voltage correction to compensate for temperature and pressure variations is performed online by the DCS.

In 2011, the detectors were exposed to about $13 \mathrm{Mhit} / \mathrm{cm}^{2}$ on average and $35 \mathrm{Mhit} / \mathrm{cm}^{2}$ for the most exposed RPCs; prototypes have been ageing-tested up to $550 \mathrm{Mhit} / \mathrm{cm}^{2}$ [10], corresponding to about ten years of safe operation in the expected running conditions.

\section{RPC performance}

\subsection{Efficiency}

The RPC efficiency can be measured from data: since the trigger algorithm requires tracks to have hits in three out of four detection planes, the efficiency of a detection element in a given plane can be obtained by using the other three planes as an external tracking system [11].

In order to check the stability of the efficiency plateau, two high voltage scans were performed within 8 months from one another. A very good reproducibility of the efficiency curve was observed (Fig. 1, left). The measured shift of the working point was less than $50 \mathrm{~V}(\simeq 0.5 \%)$ for all RPCs.

The RPC efficiency is constantly monitored in order to provide efficiency maps for offline analysis and to check its stability in time (Fig. 1, right): the average RPC efficiency, about 95\%, has been stable within $0.5 \%$ in two years of operation.

\subsection{Cluster size}

The RPC cluster size was measured for the three strip pitches employed, in both $\mathrm{pp}$ and $\mathrm{Pb}-\mathrm{Pb}$ collisions (Fig. 2).

The measured average value for strips of $2 \mathrm{~cm}$ is 1.40 , in good agreement with the value of 1.33 measured in beam tests [12] during the $R \& D$ phase. The slight difference is compatible with the fact that the FEE threshold was set to $10 \mathrm{mV}$ in the beam test and $7 \mathrm{mV}$ in the current setup. No significant difference was found between the cluster size measured in $\mathrm{pp}$ and $\mathrm{Pb}-\mathrm{Pb}$ collisions.

\subsection{Counting rate and current}

The RPC dark current and counting rate were periodically monitored (Fig. 3). The dark counting rate was measured from scalers in dedicated runs taken right after the physics fills. Its average value of $0.05 \mathrm{~Hz} / \mathrm{cm}^{2}$ is very stable in time. The spikes seen in the autumn of $2011 \mathrm{can}$ be ascribed 

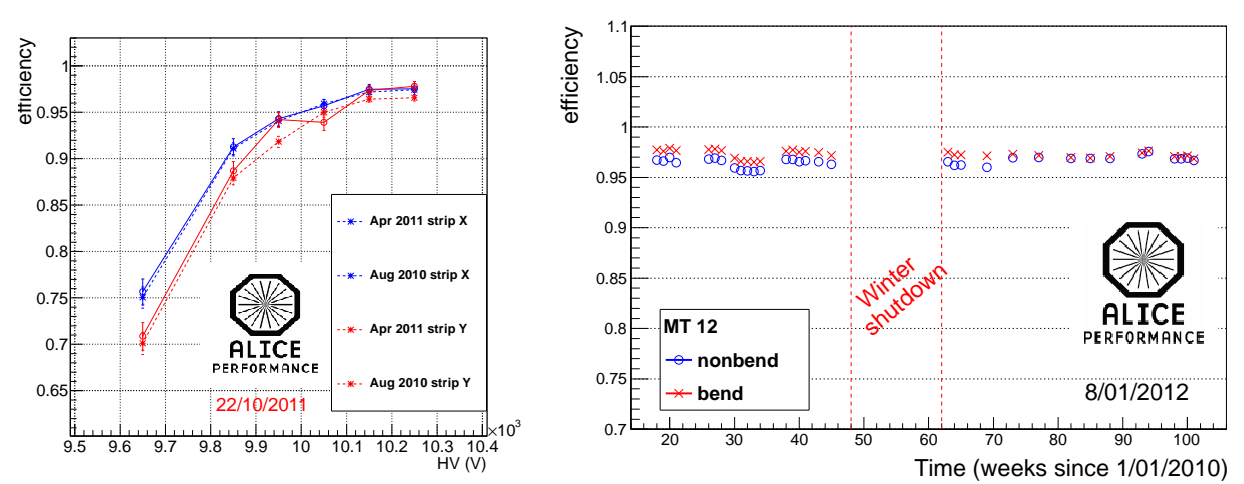

Figure 1: Left: efficiency curve of one RPC, as measured with pp collisions in two different high voltage scans 8 months apart. Right: average efficiency of one of the four detection planes (18 RPCs), as a function of time, in 2010 and 2011. The efficiency is measured separately for the X (bending) and Y (non-bending) planes.
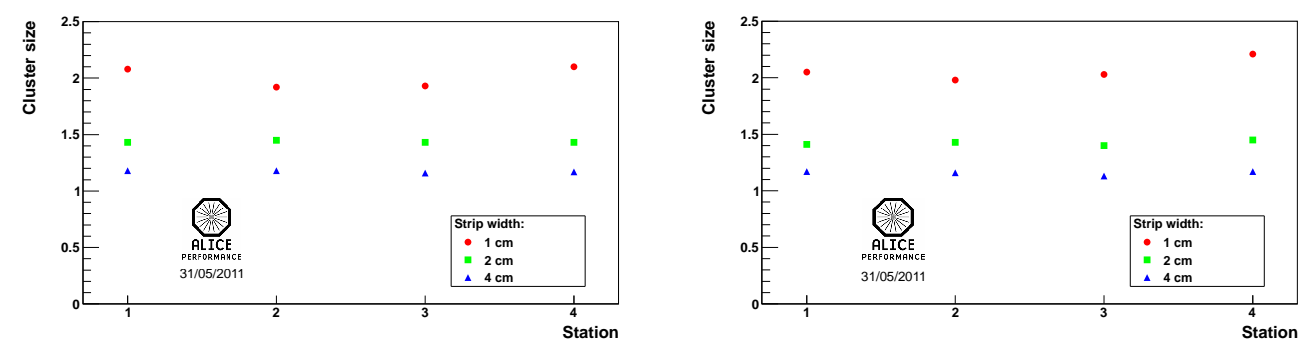

Figure 2: Average RPC cluster size for the four detection planes (18 RPC/plane), for strips with pitch 1, 2 and $4 \mathrm{~cm}$, in pp collisions at $\sqrt{s}=7 \mathrm{TeV}$ (left) and in $\mathrm{Pb}-\mathrm{Pb}$ collisions at $\sqrt{s_{N N}}=2.76 \mathrm{TeV}$ (right).

to beam-induced afterglow, since in this period the LHC proton beams reached the highest intensity [13]; in the subsequent $\mathrm{Pb}-\mathrm{Pb}$ run (November 2011), with much lower intensities, the dark rate is again very stable. The average dark current shows a slightly increasing trend, which seems to be temporarily inverted or mitigated by long periods with high voltage turned off (e.g. the winter shutdown); the average value is $1.5 \mu \mathrm{A}$, corresponding to about $0.1 \mathrm{nA} / \mathrm{cm}^{2}$.
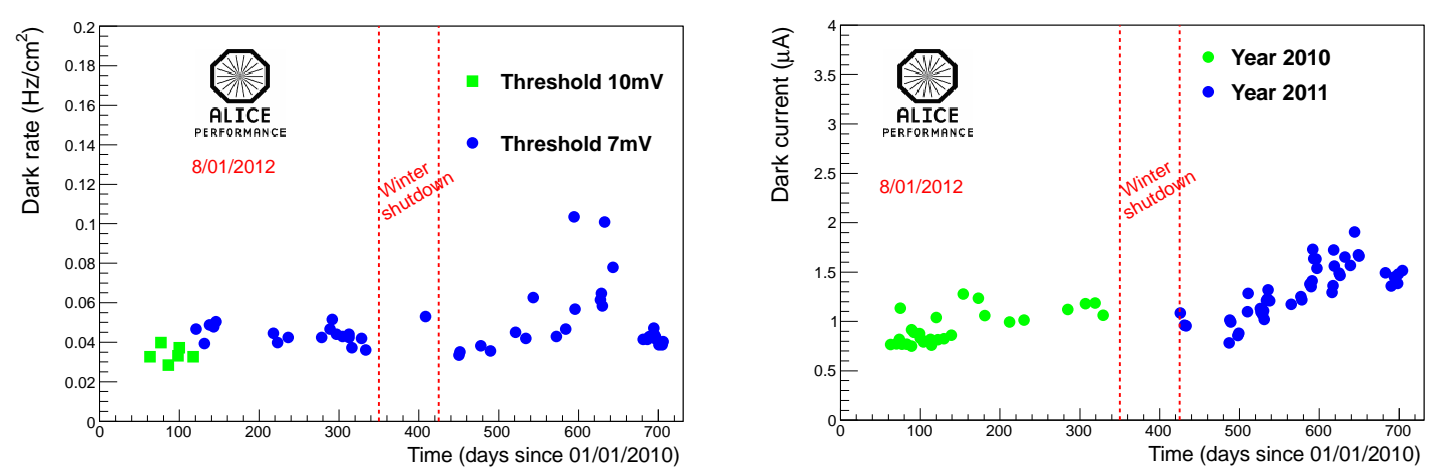

Figure 3: Average RPC dark rate (left) and dark current (right) as a function of time in 2010 and 2011. 
The maximum average current and counting rate reached during data-taking are about $10 \mathrm{~Hz} / \mathrm{cm}^{2}$ and $1 \mathrm{nA} / \mathrm{cm}^{2}$, respectively. In such conditions (autumn 2011), the RPC currents and rates are dominated by the machine-induced background [14]. Beam tests [10,12] have shown that the RPC performance is unaffected up to rates of about $80 \mathrm{~Hz} / \mathrm{cm}^{2}$.

In Figure 4, the average RPC current during physics data-taking is plotted as a function of the average counting rate: as expected, a linear correlation is found. The slope of the curve is about $2 \mu \mathrm{A} /\left(\mathrm{Hz} / \mathrm{cm}^{2}\right)$, corresponding to an average charge per hit of about $100 \mathrm{pC}$.

In Figure 5, the RPC counting rate is plotted as a function of the minimum bias trigger rate in $\mathrm{Pb}-\mathrm{Pb}$ collisions. The three curves correspond to the most exposed RPC, to the average of the RPCs on the most exposed detection plane and to the average of all RPCs. The slope of the curve corresponds to the average number of hits per minimum bias event: this quantity is $0.5 \times 10^{3} \mathrm{~cm}^{-2}$ on average and $0.8 \times 10^{3} \mathrm{~cm}^{-2}$ for the most exposed RPC. An extrapolation to $50 \mathrm{kHz}$ minimum bias rate (hypothetical scenario for an upgraded LHC [15]) leads to counting rates of $25 \mathrm{~Hz} / \mathrm{cm}^{2}$ on average and $40 \mathrm{~Hz} / \mathrm{cm}^{2}$ for the most exposed RPC. Such rates are still tolerable by the detectors in terms of rate capability. However, the detector lifetime in such a scenario might be limited. Thus, the possibility of switching to a lower-gain gas mixture in order to reduce ageing effects is being considered.
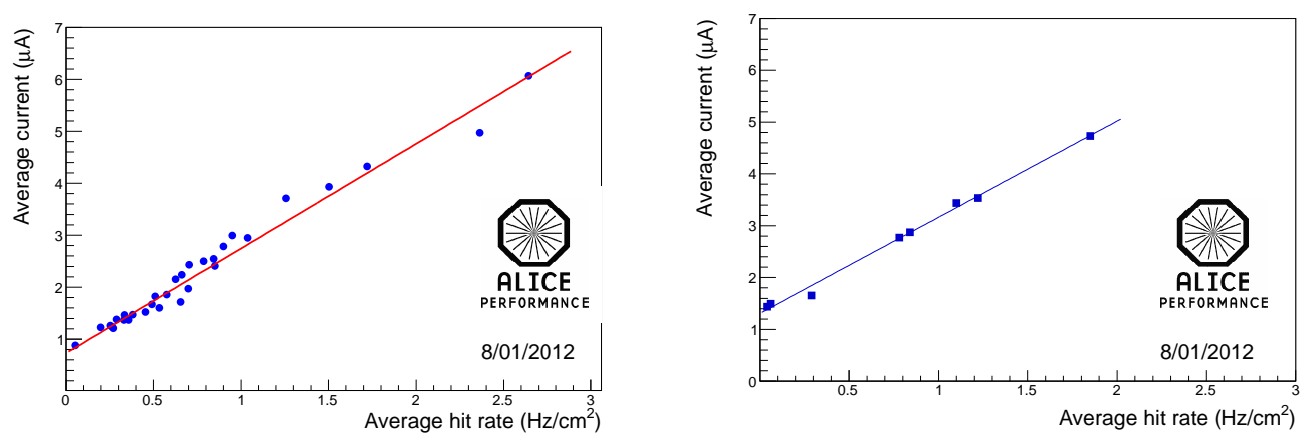

Figure 4: RPC average current as a function of the average counting rate in pp (left) and $\mathrm{Pb}-\mathrm{Pb}$ (right) collisions, with superimposed linear fit. The fitted slopes are $\left.2 \mu \mathrm{A} /\left(\mathrm{Hz}_{\mathrm{cm}}\right)^{2}\right)$ for $\mathrm{pp}$ and $\left.1.9 \mu \mathrm{A} /\left(\mathrm{Hz} / \mathrm{cm}^{2}\right)\right)$ for $\mathrm{Pb}-\mathrm{Pb}$.

\section{Conclusions}

The ALICE muon trigger system has been fully operational during the first two years of datataking at the LHC. The observed RPC performance is in agreement with the design values. The average RPC efficiency is about 95\%; the cluster size is 1.4 with $2 \mathrm{~cm}$ strips; the dark current is about $0.1 \mathrm{nA} / \mathrm{cm}^{2}$ and the dark counting rate is about $0.05 \mathrm{~Hz} / \mathrm{cm}^{2}$. The values of all these parameters are remarkably stable in time, with the sole exception of the dark current.

The ALICE muon spectrometer is playing a crucial role in the ALICE physics program, especially in the heavy flavour and quarkonia studies, in both $\mathrm{Pb}-\mathrm{Pb}[4]$ and pp $[16,17,18]$ collisions, and, also in virtue of the performance and stability of the muon trigger system, is expected to continue to do so in the next years of LHC operation. 


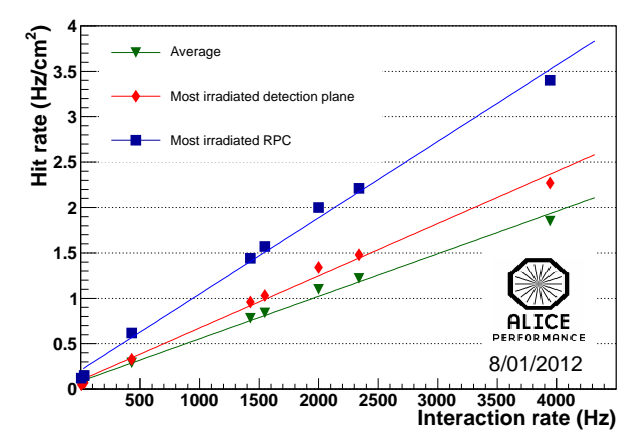

Figure 5: RPC counting rate as a function of the interaction rate in $\mathrm{Pb}-\mathrm{Pb}$ collisions: for the most exposed RPC (blue squares); average for the RPCs on the most exposed detection plane (red dots); average for all RPCs (green triangles). Lines are linear fits to the data.

\section{References}

[1] K. Aamodt et al. [ALICE Collaboration], J. Phys. G 32 (2006) 1295-2040

[2] B. Alessandro et al. [ALICE Collaboration], JINST 3 (2008) S08002

[3] Y. W. Baek, in these proceedings

[4] B. Abelev et al. [ALICE Collaboration], arXiv:1202.1383v1 [hep-ex], submitted to Phys. Rev. Lett.

[5] M. Marchisone, in these proceedings

[6] M. Gagliardi et al. [ALICE Collaboration], Nucl. Instr. Meth. A 661 S1 (2012) S45-S49

[7] A. Ferretti et al, Nucl. Instr. Meth. A 508 (2003) 106-109

[8] R. Arnaldi et al., IEEE Trans. Nucl. Sci. 52 (2005) 1176-1181

[9] M. Gagliardi et al., Nucl. Instr. Meth. A 602 (2009) 740-743

[10] F. Poggio, Ph.D. Thesis, 2006, Università degli Studi di Torino

[11] D. Stocco, ALICE-INT-2008-004

[12] F. Poggio et al., Nucl. Phys. B (Proc. Suppl.) 158 (2006) 149-153

[13] M. Ferro-Luzzi, Proceedings of the Chamonix 2012 Performance Workshop

[14] F. Bossù, Ph.D. Thesis, 2012, Università degli Studi di Torino

[15] J. Wessels et al. [ALICE Collaboration], Proceedings of the Chamonix 2012 Performance Workshop

[16] K. Aamodt et al. [ALICE Collaboration], Phys. Lett. B 704 (2011) 442-455

[17] B. Abelev et al. [ALICE Collaboration], arXiv:1203.3641v1 [hep-ex], submitted to Phys. Lett. B

[18] B. Abelev et al. [ALICE Collaboration], Phys.Rev.Lett. 108 (2012) 082001

[19] B. Abelev et al. [ALICE Collaboration], Phys. Lett. B 708 (2012) 265-275 